\title{
Determination of thermal conductivity of powders in different atmospheres by differential scanning calorimetry
}

\author{
Daniel Sánchez-Rodríguez $^{1 *}$, Joan Pere López-Olmedo ${ }^{2}$, Jordi Farjas ${ }^{1}$ and Pere Roura ${ }^{1}$ \\ ${ }^{1}$ GRMT, Dept. of Physics, University of Girona, Campus Montilivi, Edif.PII, E17071 \\ Girona, Catalonia, Spain \\ 2 UAT, ServeisTècnics de Recerca, University of Girona, Campus Montilivi, Edif. PII, \\ E17071 Girona, Catalonia, Spain \\ *Corresponding author: daniel.sanchez@udg.edu
}

\begin{abstract}
We have developed a new method to measure the thermal conductivity of powders by differential scanning calorimetry (DSC) that works with masses in amounts as low as tens of mg. The method is based on that used by Camirand to determine the thermal conductivity of materials in the form of thin sheets but introducing a hemispherical pan to contain powders in such a way that the issue of heat transfer is reduced to a one-dimensional problem. The modification of the method was successfully validated on obtaining identical results in determining the thermal conductivity of a commercial silicone with both Camirand's method and the modified method. We have also tested our method with materials that, in bulk, cover a wide range of thermal conductivities and have performed the experiments with several atmospheres and reference metals. The results are consistent with already published general trends in that they confirm that thermal conductivity of powders is mainly governed by thermal conduction through the surrounding gas.
\end{abstract}

Keywords: Thermal conductivity, powders, DSC 


\section{Introduction}

At present, the thermal conductivity of powders is commonly determined from measurements of their thermal diffusivity with the modified transient plane source method (MTPS) [1] where powders are bound inside a cylinder and heated by an axial heat source.This method, used in commercial apparatuses, requires an independent measurement of the material's heat capacity and works with a large number of powders.

Differential scanning calorimetry (DSC) is probably the most widely-used thermoanalytical technique. Among many other applications, it is used for the measurement of the heat capacity of solid or liquid substances [2] and the thermal conductivity of solids. There are various established methods to measure thermal conductivity with DSC using either the modulated temperature mode $[3,4]$ or the standard mode[5,6,7,8] but none are suitable when the sample is in the form of uncompressed powders.

Camirand [5] and, before him, Flynn and Levin [6] developed a simple method to measure the thermal conductivity of flat materials with conventional DSC, which, in this paper, we will show can be modified to measure the thermal conductivity of powders. Our method (and Camirand's method), unlike the one based on the modulated temperature mode, determines the thermal conductivity at discrete temperature values that can only correspond to the melting points of the reference metals. On the other hand, there are no limitations on the temperature range beyond those of the DSC apparatus itself, although an upper limit could be set by the crucible. Our contribution is to use a crucible containing powders in such a way that heat transfer is reduced to a one-dimensional problem. In so doing we are able to obtain the thermal resistance of powders, using just tens of milligrams of a sample, by melting a reference metal as Camirand does with sheet materials.

\section{Experimental details}

All thermal conductivity experiments were performed with a Mettler ToledoDSC822 and high purity spherical reference metals (radii in the 1-2 $\mathrm{mm}$ range) were used. A noncommercial cylindrical aluminium crucible with a hemispherical cavity (Fig. 1) with an inner radius of $2.5 \mathrm{~mm}$ was made by plastic deformation. The outer radius and height were reduced by machining until the diameter was equal to that of a standard crucible in order to avoid thermal bridges between the crucible and the apparatus. No reference pan was used. The gas flow rate was set at $40 \mathrm{mLmin}^{-1}$ and nitrogen, argon and helium, all of high purity, were selected because of their differences in thermal conductivity. A constant heating rate of $10^{\circ} \mathrm{C}$ $\min ^{-1}$ was maintained for the systematic experiments although we modified the heating rate 
from 5 to $20{ }^{\circ} \mathrm{C} \min ^{-1}$ for method validations. The DSC signal was calibrated for the three gases by measuring the area of the melting peaks.

High purity (above 99\%) commercially available alumina, iron, tin oxide and $\mathrm{Ba}$ trifluoroacetate powders were used. Particles morphology and size were very different as shown in the scanning electrons micrographs (SEM) of Fig. 2. The thermal conductivity of these materials in bulk form is set out in Table 1 . The powders were gently pressed inside the crucible.

\section{Method description}

The hemispherical crucible was completely filled with the powder being tested and a small reference metal sphere was sunk inside the powder until its centre was concentric to the crucible cavity (Fig. 1). The filled crucible was heated in the DSC apparatus at a constant heating rate until the metal melted. The slope of the melting peak, depicted in Figure 3, corresponds to the following formula:

$S_{p}=\frac{1}{R+R_{c}+R_{p}}$,

where $R, R_{c}$ and $R_{p}$ are the sensor resistance, the contact resistance between the crucible and the DSC sensor disk and the powder's resistance, respectively (see Appendix).

A second DSC curve was then recorded at the same heating rate without the powder in the crucible. The metal was flattened on the bottom of the crucible cavity to optimize the thermal contact between the metal and the crucible. Similarly to the previous case, the slope of the melting peak $\left(\mathrm{S}_{\mathrm{e}}\right)$ (Fig. 3) corresponds to the formula:

$S_{e}=\frac{1}{R+R_{c}}$.

Eqs. 1 and 2 allow the powder's thermal resistance to be obtained and the spherical symmetry makes it possible to calculate the thermal conductivity:

$\kappa=\frac{1}{2 \pi R_{p}} \cdot\left(\frac{1}{r_{i}}-\frac{1}{r_{e}}\right)$

wherer $r_{i}$ is the radius of the reference metal sphere and $r_{e}$ is the radius of the hemispherical cavity.

\section{Results}

a) Method validation

Several experiments have been performed to check the correctness and reproducibility of the method. In Table 2 we summarize the heating rates and indium sphere radii used to validate 
the method on tin oxide powders. We observe, as expected, that all of these experiments gave thermal conductivity values that were almost identical when the experimental conditions were identical except for the heating rate. The $10 \%$ deviation observed when a larger radius was tested, can be explained by an increase in the density of the powder used. Despite the independence of the heating rate, low heating rates are preferred as DSC curves usually have better defined melting slopes in these conditions.

Finally, the thermal conductivity of a commercial silicone that can be cast from a viscous state and cured at a moderate temperature of $80^{\circ} \mathrm{C}$ was measured. Once cured, this silicone remained stable beyond the melting point of indium and so was suitable to validate our method through comparison with Camirand's. A $2 \mathrm{~mm}$ thick and $5 \mathrm{~mm}$ diameter flat disk was made for that purpose. Within experimental accuracy, which was essentially limited by the thickness measurement $( \pm 0.05 \mathrm{~mm})$, we obtained the same thermal conductivity for the two methods $\left(0.103 \mathrm{~W}^{\circ} \mathrm{C}^{-1} \mathrm{~m}^{-1}\right)$. It is also worth noting that the conductivity of the silicone was similar to that of the powders (see section $b$ ). This means that we have validated our method just at the conductivity values of interest.

\section{b) Conductivity of powders}

The thermal conductivity of several powders was measured in $\mathrm{N}_{2}$ at the melting point of In $\left(156.6^{\circ} \mathrm{C}\right)$ and the results are summarized in Table 1. It is worth noting that, in contrast with their bulk counterparts, powders have very similar conductivities $( \pm 30 \%$ variation between powders) and that these are much lower than in bulk (values at $25^{\circ} \mathrm{C}$ ).

In Fig. 4 we have plotted the thermal conductivity of alumina powders for three different atmospheres at the melting points of indium, tin, lead and zinc, and have also plotted the thermal conductivity of nitrogen, argon and helium for purposes of comparison. A relative density value was maintained between 27 and 30\% for all experiments. It is important to highlight, as is seen in the figure, that there is a clear and direct relationship between the thermal conductivity of the alumina powders and the surrounding gas. The conductivity of powders increases with increased gas conductivity and temperature. In addition, the absolute values of powders are higher but similar to those of the surrounding atmosphere.

Finally, it is interesting to note that there are significant differences in the melting temperature when the reference metal is melted with and without powders contained in the crucible (Fig. 3). The relationship between this delay and the thermal conductivity of the powders will be studied in future work. 


\section{Discussion}

Since Klemensiewicz [9] reported the unexpectedly low thermal conductivities of powders, this surprising behaviour has been confirmed by many other authors $[10,11,12,13]$. All these authors have found thermal conductivities of powders that are similar to those of the surrounding gas, irrespectively of their bulk conductivity. These general trends agree with our own results as presented in Fig. 4 and Table 2.

In view of the very low thermal conductivity of powders when compared to their values in bulk (Table 1), one must conclude that the network of solid particles is very inefficient for heat transport. In the absence of any gas, the thermal resistance between particles at the points of contact must be very high. So, heat transport through the gas in the interstices between the particles is essential to understand the measured values. We can take the ratio $\kappa_{\mathrm{GAS}} / \kappa$ to qualitatively evaluate the gas contribution to heat transport. At any temperature, this ratio reaches its maximum value for $\mathrm{He}$ (Fig.4) as expected because its conductivity is the highest among the gases we have tested.

\section{Summary}

It has been demonstrated that it is possible to determine the thermal conductivity of all kinds of powders with a DSC apparatus by modifying Camirand's method. In our method, a reference metal sphere is sunk up to its equator in a hemispherical crucible completely filled with the desired powders. This method is particularly attractive as DSC is widely available and has the advantage of only requiring a small amount of mass.

We have tested our method with materials that, in bulk, cover a wide range of thermal conductivities and have performed the experiments with several gases and reference metals to evaluate the contribution of the gas. The results are consistent with the general trends already published in the literature in that they confirm that the thermal conductivity of powders is mainly governed by thermal conduction through the surrounding gas.

\section{Acknowledgements}

This work was partially funded by the Spanish Programa Nacional de Materiales through projects MAT2011-28874-C02-02 and by the Generalitat de Catalunya contract No. 2009SGR-185. The authors wish to thank the University of Girona for the PhD fellowship granted to Daniel Sánchez-Rodríguez and for the use of the thermal analysis facilities (Serveis Tècnics de Recerca). 


\section{References:}

[1] L.N. Novichenok, S.M.Ovchinniko and V.A.Marshak, "Thermal conductivity probe e.g. for complex liquids and powders - has cylinder wound alternately with heater and resistance thermometer turns" (1986) Patent n. SU1264053-A.

[2] P.Roura, D.Sanchez-Rodriguez and J.Farjas, "Measurement by differential scanning calorimetry of specific heat capacity variation due to crystallization: Application to amorphous silicon”, Thermochim.Acta 522 (2011) 161-165.

[3] S.M. Marcus and R.L. Blaine, "Thermal-conductivity of polymers, glasses and ceramics by modulated DSC", J.Therm.Anal.Calorim 243 (1994) 231-239.

[4]G.Kalogiannakis, D.Van Hemelrijck andG. van Assche,"Measurements of Thermal Properties of Carbon/Epoxy and Glass/Epoxy using Modulated Temperature Differential Scanning Calorimetry", J.Comp.Mater., 38 (2004) 163-175.

[5] C.P. Camirand, "Measurement of thermal conductivity by differential scanning calorimetry", Thermochim.Acta, 417 (2004) 1-4.

[6] J.H. Flynn and D.M. Levin, "A method for the determination of thermal conductivity of sheet materials by differential scanning calorimetry" Thermochim.Acta, 126 (1988) 93-100.

[7] I. Tavman, Y. Aydogdu, M. Kök, A. Turgut and A. Ezan "Measurement of heat capacity and thermal conductivity of HDPE/expanded graphite nanocomposites by differential scanning calorimetry" Archives of Materials Science and Engineering, 50 (2011) 56-60.

[8] G. Hakvoort, L.L.Vanreijen and A.J.Aartsen, "Measurement of the thermal conductivity of solid substances by DSC" Thermochim.Acta, 93 (1985) 317-320.

[9] Z. Klemensiewicz, “Thermal Conductivity of Powders”, Nature, 164 (1949) 589-589.

[10] L. Huang and M. S. El-Genk, "Thermal conductivity measurements of alumina powders and molded Min-K in vacuum”, Energy Convers.Manage., 42 (2001) 599-612.

[11] Y.Ming, J.Purewal, D.Liu, A.Sudik, Ch.Xu, J.Yang, M.Veenstra, K.Rhodes, R.Soltis, J.Warner, M.Gaab, U.Müller andD.J.Siegel“Thermophysical properties of MOF-5 powders”, MicroporousMesoporous Mater., 185 (2014) 235-244.

[12] B.L. Huang, Z. Ni, A. Millward, A.J.H.McGaughey, C. Uher, M. Kaviany and O. Yaghi, "Thermal conductivity of a metal-organic framework (MOF-5): Part II. Measurement”, Int. J. Heat.Mass.Transfer, 50 (2007) 405-411.

[13] R.L Mullokandov, J. Tech. Phys., U.S.S.R., 17 (1947) 1149. (Phys. Abstr., 1432 (1947)) 
Table 1.- Thermal conductivity of the powders studied in this paper at the melting point of In $\left(156.6^{\circ} \mathrm{C}\right.$ ) in $\mathrm{N}_{2}$. The bulk conductivities $\left(\right.$ at $25^{\circ} \mathrm{C}$ ) are taken from public sources

\begin{tabular}{|l|l|l|}
\hline & Thermal conductivity $/ \mathbf{W}^{\circ} \mathrm{C}^{-1} \mathbf{m}^{-1}$ \\
\hline Material & Bulk & Powders \\
\hline $\mathbf{A l}_{\mathbf{2}} \mathbf{O}_{\mathbf{3}}$ & 30 & 0.126 \\
\hline $\mathbf{F e}$ & 80.4 & 0.1 \\
\hline $\mathbf{S n O}_{2}$ & 40 & 0.059 \\
\hline $\mathbf{B a}\left(\mathbf{C F}_{\mathbf{3}} \mathbf{C O}_{2}\right)_{3}$ & 0.3 & 0.08 \\
\hline
\end{tabular}

Table 2.-Parameters and results of experiments carried out with powders in $\mathrm{N}_{2}$ at the melting pointof In ( $\beta$ is the heating rate)

\begin{tabular}{|c|c|c|c|c|c|}
\hline & $\begin{array}{l}\beta / \\
{ }^{\circ} \mathrm{Cmin}^{-1}\end{array}$ & $\begin{array}{l}\text { Metal } \\
\text { sphere } \\
\text { radius /mm }\end{array}$ & $\begin{array}{l}\text { Melting slope } \\
/ \mathrm{mW}^{\circ} \mathrm{C}^{-1}\end{array}$ & $\begin{array}{l}\text { Thermal } \\
\text { conductivity } \\
/ \mathrm{W}^{\circ} \mathrm{C}^{-1} \mathrm{~m}^{-1}\end{array}$ & $\begin{array}{l}\text { Relative } \\
\text { density } / \%\end{array}$ \\
\hline $\mathrm{SnO}_{2}$ & 5 & 1.19 & 0.9 & 0.06 & 9 \\
\hline $\mathrm{SnO}_{2}$ & 10 & 1.19 & 0.89 & 0.059 & 9 \\
\hline $\mathrm{SnO}_{2}$ & 20 & 1.19 & 0.89 & 0.059 & 9 \\
\hline $\mathrm{SnO}_{2}$ & 10 & 1.75 & 2.34 & 0.066 & 12.5 \\
\hline $\mathrm{Al}_{2} \mathrm{O}_{3}$ & 10 & 1.21 & 1.85 & 0.126 & 29.5 \\
\hline $\mathbf{F e}$ & 10 & 1.04 & 0.71 & 0.1 & 50.1 \\
\hline $\mathrm{Ba}\left(\mathrm{CF}_{\mathbf{3}} \mathrm{CO}_{2}\right)_{3}$ & 10 & 1.04 & 0.8 & 0.08 & $42 *$ \\
\hline
\end{tabular}

*assuming a theoretical density of $3.5 \mathrm{~g} / \mathrm{cm}^{3}$ 


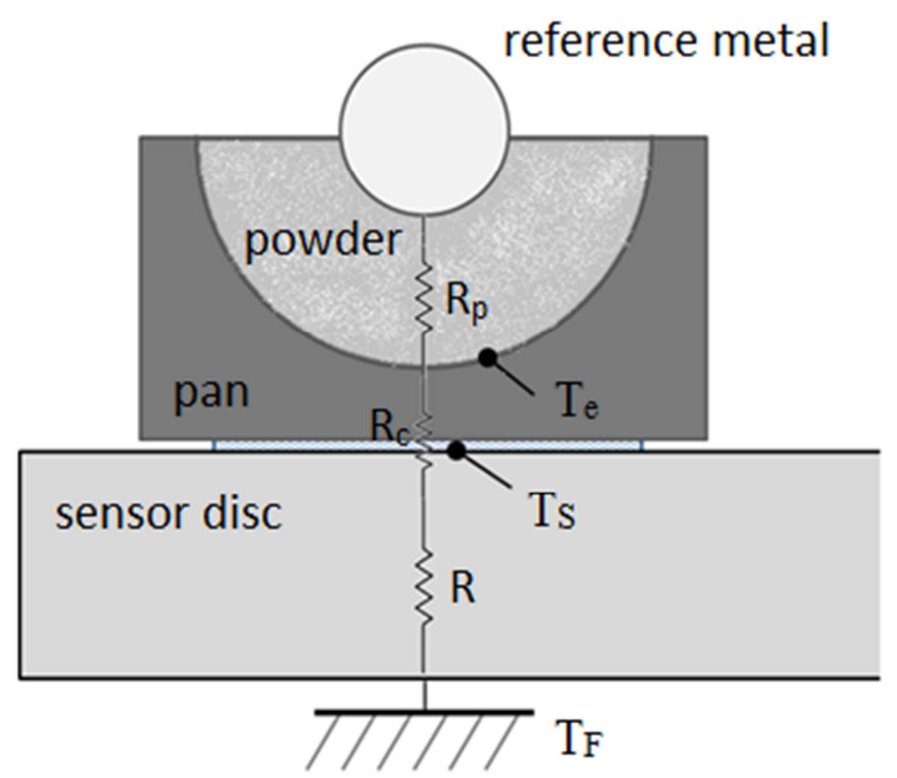

Figure 1.- Geometric appearance of the hemispherical pan placed on the sensor disk. The thermal resistances of the system are indicated (R: sensor, Rc: contact resistance, $\mathrm{Rp}$, powder) 

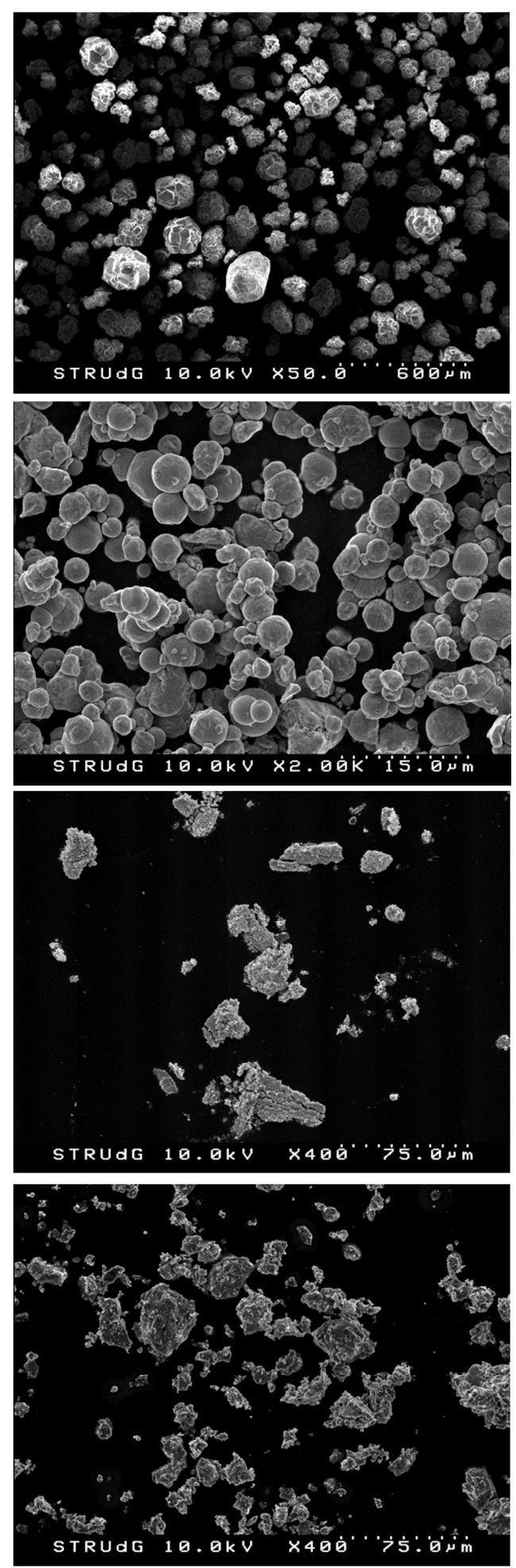

Figure 2.- From top to bottom: SEM micrographs of the alumina, iron, tin oxide and $\mathrm{Ba}(\mathrm{TFA})_{2}$ powders studied in this paper. 


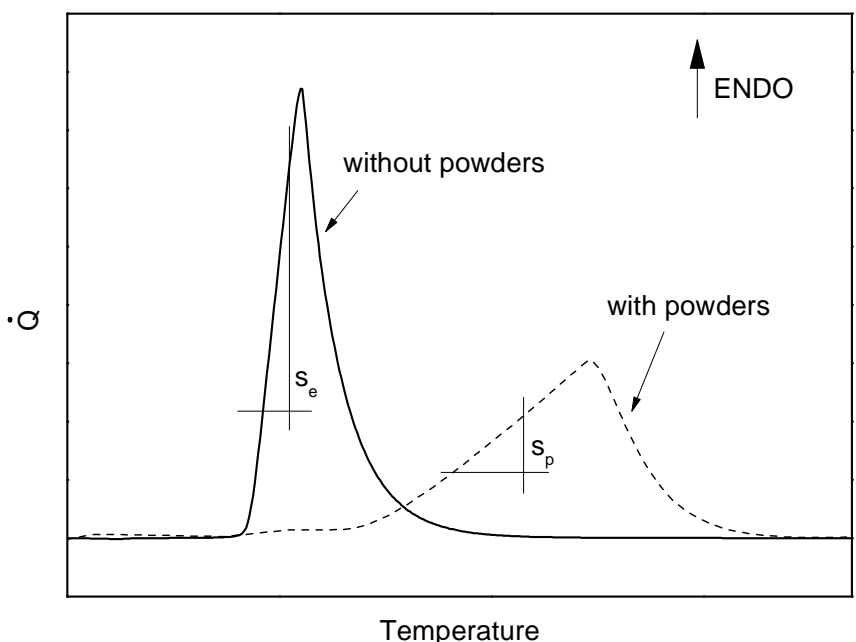

Figure 3.- Typical DSC melting curves with and without powders. The thermal resistance of the powders is obtained from the $\mathrm{S}_{\mathrm{e}}$ and $\mathrm{S}_{\mathrm{p}}$ slopes.

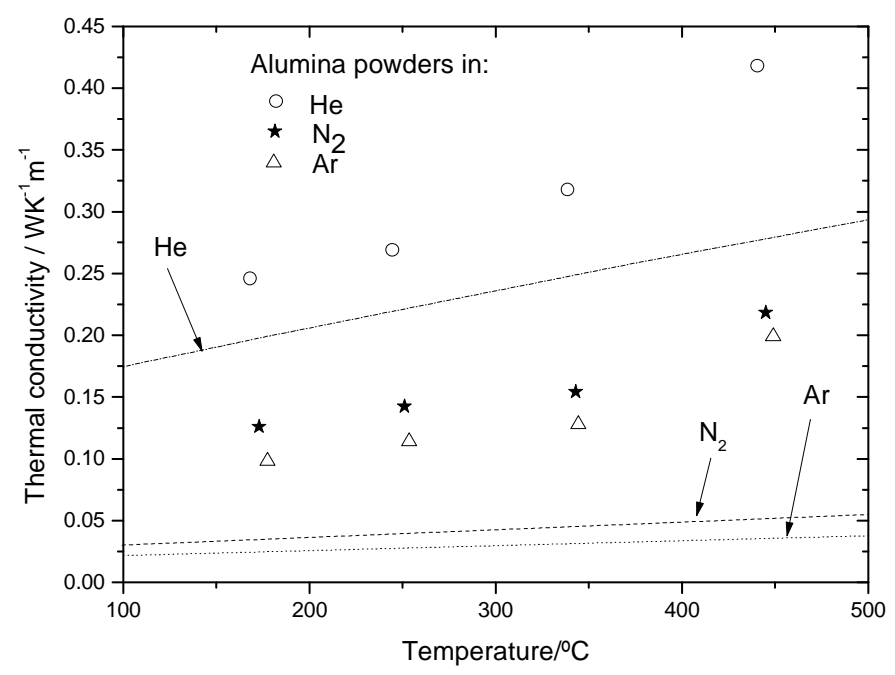

Figure 4.- Temperature dependence of the thermal conductivity of alumina powders in several gases (points). The thermal conductivity of $\mathrm{He}, \mathrm{N}_{2}$ and $\mathrm{Ar}$ are shown for comparison (lines). 


\section{Appendix: slope of the DSC signal during melting}

During melting, the metal reference remains at the melting temperature, $T_{M}$, whereas the DSC furnace is heated at a constant heating rate, $\beta$. Let us first demonstrate that any point $r$ of the powder contained in the hemispherical holder (Fig. 1) will experience a constant heating rate according to the formula:

$$
T(r, t)=f(R)+\frac{\beta_{e}}{R_{e}} R t
$$

where $t$ is time, $f$ is a time-independent function and $R$ is the thermal resistance of the powder inside the radius $r$, i.e.:

$$
R=\frac{1}{2 \pi \kappa}\left(\frac{1}{r_{i}}-\frac{1}{r}\right)
$$

where $\kappa$ is the powder thermal conductivity. Notice that the heating rate of the powder varies from zero at $r_{i}$ to its maximum value $\beta_{\mathrm{e}}$ at $r_{e}$.

We must simply verify that $\mathrm{T}(r, t)$ of Eq.(A1) satisfies the heat transport equation that, given the spherical symmetry of the system, can be written as:

$$
\frac{2}{r} \frac{\partial T}{\partial r}+\frac{\partial^{2} T}{\partial r^{2}}=\frac{\rho c}{\kappa} \frac{\partial T}{\partial t}
$$

or, substituting $r$ by $R$ in the partial derivatives:

$$
\frac{1}{2 \pi} \frac{1}{r^{4}} \frac{\partial^{2} T}{\partial R^{2}}=\rho c \frac{\partial T}{\partial t}
$$

where $\rho$ and $c$ are the density and specific heat of the powder, respectively. This verification is straightforward after the introduction of Eq.(A1) into (A4), and is left for the reader.

Once demonstrated that the powder in contact with the pan (at $r=r_{e}$ ) is heated at a constant rate, the value of $\beta_{\mathrm{e}}$ will be calculated with the assumption that we can neglect both the pan resistance and its contact resistance with the DSC sensor (i.e. $\mathrm{T}_{\mathrm{S}}=\mathrm{T}_{\mathrm{e}}-$ see Fig.1). We must simply impose a power measured by the DSC, $\dot{Q}_{\mathrm{DSC}}$ equal to the heat that enters into the powder through the pan's inner surface, $\dot{Q}_{\mathrm{e}} . \dot{Q}_{\mathrm{DSC}}$ can be easily calculated through the formula:

$$
\dot{Q}_{D S C}=\frac{T_{R E F}-T_{e}}{R},
$$

where $R$ is the thermal resistance of the DSC sensor, $\mathrm{T}_{\mathrm{REF}}$ is the reference temperature that changes at the programmed heating rate:

$$
T_{R E F}=T_{0}+\beta t
$$

and $\mathrm{T}_{\mathrm{e}}$ is given by Eq.(A1) at $\mathrm{r}=\mathrm{r}_{\mathrm{e}}$. We obtain:

$$
\dot{Q}_{D S C}=A+\frac{\beta-\beta_{e}}{R} t,
$$


where A is time-independent. On the other hand, $\dot{Q}_{e}$ is proportional to the temperature gradient at $r_{e}$ :

$$
\dot{Q}_{e}=\left.2 \pi r_{e}^{2} \kappa \frac{\partial T}{\partial r}\right|_{r e} .
$$

Introduction of Eqs.(A1) and (A2) into Eq.(A8) gives:

$$
\dot{Q}_{e}=B+\frac{\beta_{e}}{R_{e}} t
$$

where B is time-independent. Now, the condition $\dot{Q}_{D S C}=\dot{Q}_{e}$ delivers the value of $\beta_{\mathrm{e}}$ :

$$
\beta_{e}=\frac{R_{e}}{R+R_{e}} \beta
$$

And, finally, the slope of the DSC signal can be obtained after the substitution of $\beta_{\mathrm{e}}$ in Eq.(A7):

$$
\frac{d \dot{Q}_{D S C}}{d T_{R E F}}=\frac{d \dot{Q}_{D S C}}{d(\beta t)}=\frac{1}{R_{D S C}+R_{e}}
$$

If the contact resistance between the pan and the DSC sensor were not negligible, the result would be $1 / \mathrm{R}+\mathrm{R}_{\mathrm{c}}+\mathrm{R}_{\mathrm{e}}$. 